\title{
DUAL SPACE OF THE SPACE OF BOUNDED LINEAR $n$-FUNCTIONALS
}

\author{
RISTO MALCHESKI AND ZDRAVKO CVETKOVSKI
}

\begin{abstract}
In [2] are considered $n$-Banach spaces, and in [4] are considered bounded and continuous linear $n$-functionals defined on $n$-normed space and several theorems connected with them, are proved. Then is proved that: Linear $n$-functional $F$ is continuous if and only if $F$ is bounded (theorem 4). In this paper, a dual space $X^{*}$ of space of bounded linear $n$-functionals is considered and it is proved that: if $X$ is $n$-Banach space than $(X *,\|\|$.$) is Banach space.$
\end{abstract}

\section{INTRODUCTION}

Definition 1. Let $X_{i}, i=1,2, \ldots, n$ be linear subspace of same vector nnormed space. Then the mapping $F: X_{1} \times \ldots \times X_{n} \rightarrow \mathbb{R}$ is called $n$ functional with domain $X_{1} \times X_{2} \times \ldots \times X_{n}$.

Definition 2. Let $F$ be nfunctional with domain $X_{1} \times X_{2} \times \ldots \times X_{n}$. Then $F$ is linear $n$ - functional if the following conditions are satisfied:

1. $F\left(x_{1}+y_{1}, x_{2}+y_{2}, \ldots, x_{n}+y_{n}\right)=\sum_{\substack{z_{i} \in\left\{x_{i}, y_{i}\right\} \\ i=1, \ldots, n}} F\left(z_{1}, z_{2}, \ldots, z_{n}\right)$

2. $F\left(\alpha_{1} x_{1}, \alpha_{2} x_{2}, \ldots, \alpha_{n} x_{n}\right)=\alpha_{1} \alpha_{2} \ldots \alpha_{n} F\left(x_{1}, x_{2}, \ldots, x_{n}\right)$

$\alpha_{i} \in \mathbb{R}, i=1,2, \ldots, n$

Definition 3. Let $X$ be n-normed space. Let $F$ be n-functional with domain $D(F) \subseteq X^{n}$ then $F$ is bounded if there exists real number $K \geqslant 0$ such that $F\left(\alpha_{1} x_{1}, \alpha_{2} x_{2}, \ldots, \alpha_{n} x_{n}\right)=\alpha_{1} \alpha_{2} \ldots \alpha_{n} F\left(x_{1}, x_{2}, \ldots, x_{n}\right)$.

Let $F$ be bounded $n$-functional, we define norm of $F$, denoted by $\|F\|$, with

$\|F\|=\inf \left\{K\left\|F\left(x_{1}, x_{2}, \ldots, x_{n}\right) \mid \leqslant K\right\| x_{1}, x_{2}, \ldots, x_{n} \|,\left(x_{1}, x_{2}, \ldots, x_{n}\right) \in D(F)\right\}$

If $F$ is unbounded $n$-functional, then we define $\|F\|=+\infty$. 
In this context for bounded linear n-functionals in [4] the following properties are proved.

Lemma 1. Let $F$ be a bounded linear $n$-functional and $x_{i}, i=1, \ldots, n$, are linearly dependent vectors such that $\left(x_{1}, x_{2}, \ldots, x_{n}\right) \in D(F)$. Then $F\left(x_{1}, x_{2}, \ldots, x_{n}\right)=0$.

Theorem 1. Let $F$ be a bounded linear n-functional on domain $D(F)$. Then

$$
\begin{aligned}
& \|F\|=\sup \left\{\left|F\left(x_{1}, x_{2}, \ldots, x_{n}\right)\right| ;\left\|x_{1}, x_{2}, \ldots, x_{n}\right\|=1,\left(x_{1}, x_{2}, \ldots, x_{n}\right) \in D(F)\right\} \\
& =\sup \left\{\frac{\left|F\left(x_{1}, x_{2}, \ldots, x_{n}\right)\right|}{\left\|x_{1}, x_{2}, \ldots, x_{n}\right\|} ;\left\|x_{1}, x_{2}, \ldots, x_{n}\right\| \neq 0,\left(x_{1}, x_{2}, \ldots, x_{n}\right) \in D(F)\right\} .
\end{aligned}
$$

Further on, continuity of linear $n$-functional is defined as following.

Definition 4. Let $F$ be n-functional. Then $F$ is continuous at the point $\left(x_{1}, x_{2}, \ldots, x_{n}\right)$ if for all $\varepsilon>0$ exist $\delta>0$ such that

$$
\left|F\left(x_{1}, x_{2}, \ldots, x_{n}\right)-F\left(y_{1}, y_{2}, \ldots, y_{n}\right)\right|<\varepsilon
$$

always when

$$
\left\|z_{1 j}, z_{2 j}, \ldots, z_{n j}\right\|<\delta
$$

where

$$
z_{i j}=\left\{\begin{array}{l}
x_{i}-y_{i}, i=j \\
x_{i} \vee y_{i}, i \neq j
\end{array}\right.
$$

for $j=1,2, \ldots, n$. The $n$-functional $F$ is continuous if $F$ is continuous at every point from its domain.

In [4], for continuous $n$-functionals are proved the following properties.

Theorem 2. If the linear $n$-functional $F$ is continuous at the point $(0,0, \ldots, 0)$, then $F$ is continuous at every point from its domain $D(F)$.

Theorem 3. Linear $n$-functional $F$ is continuous if and only if $F$ is bounded.

Definition 5. The sequence $\left\{x_{k}\right\}$ from the vector $n$-normed space $L$ is Cauchy sequence if there exists linear independent vectors $y_{1}, y_{2}, \ldots, y_{n}$ such that

$$
\begin{aligned}
& \lim _{k, m \rightarrow \infty}\left\|x_{k}-x_{m}, y_{2}, \ldots, y_{n-1}, y_{n}\right\|=0 \\
& \lim _{k, m \rightarrow \infty}\left\|x_{k}-x_{m}, y_{1}, \ldots, y_{i-1}, y_{i+1}, \ldots, y_{n}\right\|=0, \quad i=2, \ldots, n-1 \\
& \lim _{k, m \rightarrow \infty}\left\|x_{k}-x_{m}, y_{1}, \ldots, y_{n-1}\right\|=0 .
\end{aligned}
$$


Definition 6. The sequence $\left\{x_{k}\right\}$ from n-normed space $L$ is convergent if there exist $x \in L$ such that

$\lim _{k \rightarrow \infty}\left\|x_{k}-x, y_{1}, \ldots, y_{n-1}\right\|=0$, for all $y_{1}, y_{2}, \ldots, y_{n-1} \in L$.

For $x$ we shall say that is limit for the sequence $\left\{x_{k}\right\}$ and we'll write $x_{k} \rightarrow x, k \rightarrow \infty$.

Definition 7. For n-normed space $L$, well say that is $n$-Banach space if every Cauchy sequence is convergent.

In [4] the following property is proved.

Theorem 4. Every real n-normed vector space with dimension $n$ is $n$ Banach space.

\section{DUAL SPACE OF THE SPACE OF BOUNDED LINEAR $n$-FUCTIONALS}

Definition 8. Let $X$ be $n$-Banach space, $X^{*}$ is a set of bounded linear $n$-functionals on domain $X^{n}$ and let $F, G \in X^{*}$. We define

a) $F=G$ if $F\left(x_{1}, x_{2}, \ldots, x_{n}\right)=G\left(x_{1}, x_{2}, \ldots, x_{n}\right)$, for all $\left(x_{1}, x_{2}, \ldots, x_{n}\right) \in$ $X^{n}$,

b) $(F+G)\left(x_{1}, x_{2}, \ldots, x_{n}\right)=F\left(x_{1}, x_{2}, \ldots, x_{n}\right)+G\left(x_{1}, x_{2}, \ldots, x_{n}\right)$, for all $\left(x_{1}, x_{2}, \ldots, x_{n}\right) \in X^{n}$,

c) $(\alpha F)\left(x_{1}, x_{2}, \ldots, x_{n}\right)=\alpha F\left(x_{1}, x_{2}, \ldots, x_{n}\right)$, for all $\alpha$ and all $\left(x_{1}, x_{2}, \ldots, x_{n}\right) \in X^{n}$.

Theorem 5. Let $X$ be $n$-Banach space. Then $\left(X^{*},\|\|.\right)$ is Banach space.

Proof. Let $\left(x_{1}, x_{2}, \ldots, x_{n}\right),\left(y_{1}, y_{2}, \ldots, y_{n}\right) \in X^{n}$ and $\alpha_{i} \in \mathbb{R}, i=1,2, \ldots, n$. Then according to Definition 2. we have

$$
\begin{aligned}
& (F+G)\left(x_{1}+y_{1}, x_{2}+y_{2}, \ldots, x_{n}+y_{n}\right)= \\
& =F\left(x_{1}+y_{1}, x_{2}+y_{2}, \ldots, x_{n}+y_{n}\right)+G\left(x_{1}+y_{1}, x_{2}+y_{2}, \ldots, x_{n}+y_{n}\right)= \\
& =\sum_{\substack{z_{i} \in\left\{x_{i}, y_{i}\right\} \\
i=1,2, \ldots, n}} F\left(z_{1}, z_{2}, \ldots, z_{n}\right)+\sum_{\substack{z_{i} \in\left\{x_{i}, y_{i}\right\} \\
i=1,2, \ldots, n}} G\left(z_{1}, z_{2}, \ldots, z_{n}\right)= \\
& =\sum_{\substack{z_{i} \in\left\{x_{i}, y_{i}\right\} \\
i=1,2, \ldots, n}}(F+G)\left(z_{1}, z_{2}, \ldots, z_{n}\right) \\
& \quad(F+G)\left(\alpha_{1} x_{1}, \alpha_{2} x_{2}, \ldots, \alpha_{n} x_{n}\right)= \\
& \quad=F\left(\alpha_{1} x_{1}, \alpha_{2} x_{2}, \ldots, \alpha_{n} x_{n}\right)+G\left(\alpha_{1} x_{1}, \alpha_{2} x_{2}, \ldots, \alpha_{n} x_{n}\right) \\
& \quad=\alpha_{1} \alpha_{2} \ldots \alpha_{n} F\left(x_{1}, x_{2}, \ldots, x_{n}\right)+\alpha_{1} \alpha_{2} \ldots \alpha_{n} G\left(x_{1}, x_{2}, \ldots, x_{n}\right) \\
& \quad=\alpha_{1} \alpha_{2} \ldots \alpha_{n}\left[F\left(x_{1}, x_{2}, \ldots, x_{n}\right)+G\left(x_{1}, x_{2}, \ldots, x_{n}\right)\right] \\
& \quad=\alpha_{1} \alpha_{2} \ldots \alpha_{n}(F+G)\left(x_{1}, x_{2}, \ldots, x_{n}\right) .
\end{aligned}
$$


Further on, because of Definition 3 we have

$$
\begin{aligned}
\mid(F+G)\left(x_{1}, x_{2}, \ldots\right. & \left., x_{n}\right)|=| F\left(x_{1}, x_{2}, \ldots, x_{n}\right)+G\left(x_{1}, x_{2}, \ldots, x_{n}\right) \mid \\
& \leq\left|F\left(x_{1}, x_{2}, \ldots, x_{n}\right)\right|+\left|G\left(x_{1}, x_{2}, \ldots, x_{n}\right)\right| \\
& \leq\|F\| \cdot\left\|x_{1}, x_{2}, \ldots, x_{n}\right\|+\|G\| \cdot\left\|x_{1}, x_{2}, \ldots, x_{n}\right\| \\
& =(\|F\|+\|G\|)\left\|x_{1}, x_{2}, \ldots, x_{n}\right\|,
\end{aligned}
$$

which means that $F+G \in X^{*}$ and clearly $\|F+G\| \leq\|F\|+\|G\|$.

Analogously we can prove that for every $\alpha$ and every $F \in X^{*}, \alpha F \in X^{*}$ and $\|\alpha F\|=|\alpha| \cdot|| F||$ holds.

From the other hand, according to Definition 3 we have $\left|F\left(x_{1}, x_{2}, \ldots, x_{n}\right)\right| \leq\|F\| \cdot\left\|x_{1}, x_{2}, \ldots, x_{n}\right\|$, for all $\left(x_{1}, x_{2}, \ldots, x_{n}\right) \in X^{n}$, so $\|F\|=0$ if and only if $F=0$, which means that $X^{*}$ is vector space with norm defined by (1).

Let $\left\{F_{k}\right\}$ be Cauchy sequence on $X *$, i.e. let

$$
\lim _{\substack{m \rightarrow \infty \\ k \rightarrow \infty}}\left\|F_{k}-F_{m}\right\|=0
$$

Then for all $\left(x_{1}, x_{2}, \ldots, x_{n}\right) \in X^{n}$ is true that

$$
\left|F_{k}\left(x_{1}, x_{2}, \ldots, x_{n}\right)-F_{m}\left(x_{1}, x_{2}, \ldots, x_{n}\right)\right| \leq\left\|F_{k}-F_{m}\right\| \cdot\left\|x_{1}, x_{2}, \ldots, x_{n}\right\|
$$

which means that for every $\left(x_{1}, x_{2}, \ldots, x_{n}\right) \in X^{n}$ the real sequence $\left\{F_{k}\left(x_{1}, x_{2}, \ldots, x_{n}\right)\right\}$ is a Cauchy sequence. On $X^{n}$ let define functional $F$ with

$$
F\left(x_{1}, x_{2}, \ldots, x_{n}\right)=\lim _{k \rightarrow \infty} F_{k}\left(x_{1}, x_{2}, \ldots, x_{n}\right),\left(x_{1}, x_{2}, \ldots, x_{n}\right) \in X^{n} .
$$

Then, for all $\left(x_{1}, x_{2}, \ldots, x_{n}\right),\left(y_{1}, y_{2}, \ldots, y_{n}\right) \in X^{n}$ and $\alpha_{i} \in \mathbb{R}, i=$ $1,2, \ldots, n$ we have

$$
\begin{aligned}
& F\left(x_{1}+y_{1}, x_{2}+y_{2}, \ldots, x_{n}+y_{n}\right)=\lim _{k \rightarrow \infty} F_{k}\left(x_{1}+y_{1}, x_{2}+y_{2}, \ldots, x_{n}+y_{n}\right) \\
& =\lim _{k \rightarrow \infty} \sum_{\substack{z_{i} \in\left\{x_{i}, y_{i}\right\} \\
i=1, \ldots, n}} F_{k}\left(z_{1}, z_{2}, \ldots, z_{n}\right)=\sum_{\substack{z_{i} \in\left\{x_{i}, y_{i}\right\} \\
i=1, \ldots, n}} \lim _{k \rightarrow \infty} F_{k}\left(z_{1}, z_{2}, \ldots, z_{n}\right)= \\
& =\sum_{\substack{z_{i} \in\left\{x_{i}, y_{i}\right\} \\
i=1, \ldots, n}} F\left(z_{1}, z_{2}, \ldots, z_{n}\right)
\end{aligned}
$$

and

$$
\begin{aligned}
F\left(\alpha_{1} x_{1}, \alpha_{2} x_{2}, \ldots, \alpha_{n} x_{n}\right) & =\lim _{k \rightarrow \infty} F_{k}\left(\alpha_{1} x_{1}, \alpha_{2} x_{2}, \ldots, \alpha_{n} x_{n}\right) \\
= & \lim _{k \rightarrow \infty} \alpha_{1} \alpha_{2} \ldots \alpha_{n} F_{k}\left(x_{1}, x_{2}, \ldots, x_{n}\right) \\
= & \alpha_{1} \alpha_{2} \ldots \alpha_{n} \lim _{k \rightarrow \infty} F_{k}\left(x_{1}, x_{2}, \ldots, x_{n}\right) \\
= & \alpha_{1} \alpha_{2} \ldots \alpha_{n} F\left(x_{1}, x_{2}, \ldots, x_{n}\right),
\end{aligned}
$$


i.e. $F$ is nlinear functional. On the other hand, for the sequence $\left\{F_{k}\right\}$, $\left|\left\|F_{k}\right\|-\left\|F_{m}\right\|\right| \leq\left\|F_{k}-F_{m}\right\|$ holds.

Now from (2) we get that $\left\{\left\|F_{k}\right\|\right\}$ is real Cauchy sequence, which means that there exist $K \in \mathbb{R}$ such that $\left\|F_{k}\right\| \leqslant K$, for all $k \in \mathbb{N}$, from where we get

i.e. $F \in X *$.

$$
\begin{aligned}
\left|F\left(x_{1}, x_{2}, \ldots, x_{n}\right)\right| & =\left|\limsup _{k \rightarrow \infty} F_{k}\left(x_{1}, x_{2}, \ldots, x_{n}\right)\right| \\
& =\limsup _{k \rightarrow \infty}\left|F_{k}\left(x_{1}, x_{2}, \ldots, x_{n}\right)\right| \\
& \leq \limsup _{k \rightarrow \infty}|| F_{k}\|\cdot\| x_{1}, x_{2}, \ldots, x_{n} \| \\
& \leq K\left\|x_{1}, x_{2}, \ldots, x_{n}\right\|,
\end{aligned}
$$

We'll prove that $\left\{F_{k}\right\}$ converges to $F$. Let $\left\|x_{1}, x_{2}, \ldots, x_{n}\right\| \neq 0$. If $\varepsilon>0$ is chosen, then from (2) we have that there exist $n_{0} \in \mathbb{N}$ such that $\left\|F_{m}-F_{k}\right\|<\varepsilon$ when $m, k>n_{0}$, so by Definition 3 we have

$$
\begin{gathered}
\left|F_{m}\left(x_{1}, x_{2}, \ldots, x_{n}\right)-F_{k}\left(x_{1}, x_{2}, \ldots, x_{n}\right)\right| \leq\left\|F_{m}-F_{k}\right\| \cdot\left\|x_{1}, x_{2}, \ldots, x_{n}\right\| \\
\leq \varepsilon\left\|x_{1}, x_{2}, \ldots, x_{n}\right\|,
\end{gathered}
$$

for all $m, k \geq n_{0}$. On the other hand, because of

$$
F\left(x_{1}, x_{2}, \ldots, x_{n}\right)=\lim _{k \rightarrow \infty} F_{k}\left(x_{1}, x_{2}, \ldots, x_{n}\right)
$$

there exist $M=M\left(x_{1}, x_{2}, \ldots, x_{n}\right)>n_{0}$ such that

$$
\left|F_{M}\left(x_{1}, x_{2}, \ldots, x_{n}\right)-F\left(x_{1}, x_{2}, \ldots, x_{n}\right)\right|<\varepsilon\left\|x_{1}, x_{2}, \ldots, x_{n}\right\| .
$$

So we have

$$
\begin{aligned}
& \left|F_{k}\left(x_{1}, x_{2}, \ldots, x_{n}\right)-F\left(x_{1}, x_{2}, \ldots, x_{n}\right)\right| \leq \\
& \leq\left|F_{k}\left(x_{1}, x_{2}, \ldots, x_{n}\right)-F_{M}\left(x_{1}, x_{2}, \ldots, x_{n}\right)\right|+ \\
& \quad+\left|F_{M}\left(x_{1}, x_{2}, \ldots, x_{n}\right)-F\left(x_{1}, x_{2}, \ldots, x_{n}\right)\right| \\
& \leq \varepsilon|| x_{1}, x_{2}, \ldots, x_{n}\|+\varepsilon\| x_{1}, x_{2}, \ldots, x_{n}\|=2 \cdot \varepsilon\| x_{1}, x_{2}, \ldots, x_{n} \|
\end{aligned}
$$

for $k>n_{0}$. If $\left\|x_{1}, x_{2}, \ldots, x_{n}\right\|=0$, then the vectors $x_{1}, x_{2}, \ldots, x_{n}$ are linearly dependent, and according to Lema 1 it follows that

$$
F_{k}\left(x_{1}, x_{2}, \ldots, x_{n}\right)=0=F\left(x_{1}, x_{2}, \ldots, x_{n}\right)
$$

which means $\left|F_{k}\left(x_{1}, x_{2}, \ldots, x_{n}\right)-F\left(x_{1}, x_{2}, \ldots, x_{n}\right)\right| \leq 2 \cdot \varepsilon\left\|x_{1}, x_{2}, \ldots, x_{n}\right\|$, for all $k>n_{0}$. Hence, for all $\left(x_{1}, x_{2}, \ldots, x_{n}\right) \in X^{n}$ the following holds $\left|F_{k}\left(x_{1}, x_{2}, \ldots, x_{n}\right)-F\left(x_{1}, x_{2}, \ldots, x_{n}\right)\right| \leq 2 \cdot \varepsilon|| x_{1}, x_{2}, \ldots, x_{n} \|$, for all $k>n_{0}$. i.e. accordingly to Definition 3 we get $\left\|F_{k}-F\right\| \leq 2 \varepsilon$, for $k>n_{0}$, i.e. $\left\{F_{k}\right\}$ converge to $F$.

Finally from the arbitrarily of the Cauchy sequence $\left\{F_{k}\right\}$ we have that $(X *,\|\|$.$) is Banach space.$ 


\section{REFERENCES}

[1] Kurepa S.: Funkcionalna analiza, Skolska knjiga, Zagreb

[2] Малчески Р., Малчески А.: n-банахови простори, Зборник на трудови од II конгерс на математичарите и информатичарите на македонија, Охрид (2000)

[3] Misiak A.: $n$-Inner Product Spaces, Math.Nachr. 140

[4] Чаламани С., Малчески Р.: Непрекинати линеарни $n$-функционали, Зборник на трудови од III конгрес на СMМ, (2008) 
DUAL SPACE OF THE SPACE OF BOUNDED LINEAR $n$-FUNCTIONALS

\section{ДУАЛЕН ПРОСТОР НА ПРОСТОРОТ ОГРАНИЧЕНИ ЛИНЕАРНИ $n$ - ФУНКЦИОНАЛИ}

Ристо Малчески, Здравко Цветковски

\section{Р е з и м е}

Во [2] се разгледани $n$-банаховите простори, а во [4] се разгледани ограничените и непрекинатите линеарни $n$-функционали дефинирани на $n$-нормиран простор и се докажани неколку тврдења во врска со истите. Притоа, е докажано дека: Линеарниот $n$-функционал $F$ е непрекинат ако и само ако е ограничен (теорема 4). Во оваа работа е разгледан дуалниот простор $X^{*}$ на просторот ограничени линеарни $n$-функционали и е докажано дека ако $X$ е $n$-банахов простор, тогаш $\left(X^{*},\|\cdot\|\right)$ е Банахов простор.

FACUlty for informatics, FON University, Skopje, Macedonia

E-mail address: risto.malceski@gmail.com

Faculty for informatics, European University, Skopje, Macedonia

E-mail address: zdrcvet@yahoo.com 